\title{
Clinical diagnosis and detection of genetic mutations of pancreatic metastases: A report of four cases and review of the literature
}

\author{
YE LI $^{1,2^{*}}$, ZIXIANG ZHANG ${ }^{1,2^{*}}$, YI ZHANG ${ }^{1,2}$, JIAN YANG $^{1,2}$, \\ DONGMING ZHU ${ }^{1,2}$, DECHUN LI ${ }^{1,2}$ and JIAN ZHOU ${ }^{1,2}$ \\ ${ }^{1}$ Department of General Surgery and ${ }^{2}$ Pancreatic Disease Research Center, The First \\ Affiliated Hospital of Soochow University, Suzhou, Jiangsu 215006, P.R. China
}

Received January 5, 2017; Accepted March 21, 2017

DOI: $10.3892 / 01.2017 .6260$

\begin{abstract}
The present study aimed to report our clinical experience regarding the diagnosis and detection of genetic mutations of pancreatic metastases, and to review the relevant literature to expand knowledge of this disease. A total of 4 cases involving pancreatic metastases, which were treated at The First Affiliated Hospital of Soochow University between January 2013 and July 2016, were retrospectively analyzed. This retrospective study considered the clinicopathological variables of the 4 patients, and compared this data with those from the literature, which was searched using PubMed, EMBASE and the Cochrane Library. All 4 patients with pancreatic metastases were diagnosed by computed tomography (CT) scan and confirmed by pathological staining and immunohistochemistry. Mutation analysis was performed in 3 patients to obtain precise mutation information for guiding and evaluating the use of molecularly targeted drugs. In summary, pancreatic metastases are rare and the majority of pancreatic metastases develop from renal cell carcinoma. Diagnoses of pancreatic metastases predominantly rely on $\mathrm{CT}$, pathology and immunohistochemistry. Detection of mutations has clinical value in auxiliary diagnosis and therapy of pancreatic metastases. Based on mutation information, molecularly targeted drugs may prolong the survival of patients with unresectable pancreatic metastases.
\end{abstract}

\section{Introduction}

Pancreatic metastases are rare. Metastases are reported to account for $2-5 \%$ of all malignant lesions occurring in the

Correspondence to: Dr Jian Zhou, Department of General Surgery, The First Affiliated Hospital of Soochow University, 188 Shizi Street, Suzhou, Jiangsu 215006, P.R. China

E-mail: zhoujian06@suda.edu.cn

*Contributed equally

Key words: pancreatic metastases, diagnosis, genetic mutation detection, pancreatic metastasectomy pancreas (1-5). Studies have reported that people of $\sim 60$ years old are at high risk of developing pancreatic metastases (1-3). Metastases to the pancreas may occur a significant amount of time subsequent to diagnosis and management of the primary tumor (3). Primary tumors that may give rise to pancreatic metastases include renal cell carcinoma (RCC), non-small cell lung carcinoma, melanoma, sarcoma and colorectal carcinoma. Previous studies have indicated that the kidney may be more common than the lung as a primary tumor site of metastases to the pancreas $(1,4)$. Symptoms of pancreatic metastases are often not apparent, and these lesions are thus detected incidentally by physical examination in the majority of cases (6). Abdominal computed tomography (CT) scan and magnetic resonance imaging (MRI) examinations can be used in the differential diagnosis of pancreatic lesions (7). Pathological analysis is the gold standard in the diagnosis of pancreatic metastases (8). However, occasionally it is difficult to diagnose pancreatic metastasis based on pathology alone (9).

In recent years, pancreatic metastases have been observed with increasing frequency, particularly at high-volume pancreatic surgery centers, due to increased utilization of advanced imaging methods, including ultrasonography, CT, MRI and endoscopic ultrasonography (EUS) (10). However, the treatment for pancreatic metastases remains controversial. The majority of patients with pancreatic metastases are in the advanced stages of primary disease (11); however, the surgical resection of pancreatic metastases may still confer a survival benefit, based on a number of small, retrospective case studies, as previously reviewed (5).

To the best of our knowledge, the association between genetic mutations and pancreatic metastases is not well-reported. The present study aimed to report our clinical experience regarding the diagnosis and detection of mutations in cases of pancreatic metastases, and to review the relevant literature, in order to expand the knowledge pertaining to this disease.

\section{Patients and methods}

Patients. A retrospective analysis of the history and clinicopathological features of patients with pancreatic metastases was performed. Between January 2013 and July 2016, 394 cases of pancreatic-occupying lesions were documented 
in the database of The First Affiliated Hospital of Soochow University (Suzhou, China), of which 4 cases were diagnosed as pancreatic metastases.

A number of different variables were reviewed for each case, including age, symptoms, tumor focality and size, tumor location, tumor pathological types, type of primary tumor, latency period between primary tumor and metastases, and data regarding peri-pancreatic infiltration, lymph node metastases, extra-pancreatic metastases, mutations, and treatment methods.

The study was approved by the ethics committee of The First Affiliated Hospital of Soochow University, and all 4 patients provided written informed consent.

Diagnosis. The following methods were used in the diagnosis of pancreatic metastases: CT, pathological staining, immunohistochemistry and mutation detection. For the immunohistochemical analyses, adjacent non-tumor pancreatic tissues were used as the normal control group, and are shown for Cases 1, 2 and 3. Case 4 underwent EUS-guided fine-needle aspiration (FNA), which did not include non-tumor tissues.

Literature search strategy. Studies were identified by searching PubMed (https://www.ncbi.nlm.nih.gov/pubmed), EMBASE (https://www.embase.com) and the Cochrane Library (http://www.cochranelibrary.com). The search term was 'pancreatic metastases'. No language limitation was applied. When a study reporting the same patient cohort was included in several publications, only the most recent or complete study was selected.

Pathological hematoxylin and eosin staining and immunohistochemistry. Samples were fixed with $10 \%$ formalin overnight at room temperature (GE Healthcare Life Sciences, Logan, UT, USA) and embedded in paraffin (GE Healthcare Life Sciences) and sectioned. Serial sections (4- $\mu \mathrm{m})$ were stained with hematoxylin-eosin (Muto Pure Chemicals Co. Ltd, Tokyo, Japan) or subjected to immunohistological staining. Immunohistological staining: Subsequent to placing in blocking reagent (3\% bovine serum albumin; Roche Diagnostics, Basel, Switzerland) for $15 \mathrm{~min}$ at room temperature, the sections were incubated with primary mouse monoclonal anti-Ki-67 (dilution, 1:500; cat. no., ab6526; Abcam, Cambridge, MA, USA) or mouse polyclonal anti-cytokeratin (dilution, 1:500; cat. no., ab9377; Abcam) antibody overnight at $4^{\circ} \mathrm{C}$, followed by incubation with horseradish peroxidase-conjugated polyclonal goat anti-mouse or anti-rabbit IgG secondary antibody (dilution, 1:500; cat. no., ab97200; Abcam) for $2 \mathrm{~h}$ at $4^{\circ} \mathrm{C}$. The signal was visualized by treatment with 3,3'-diaminobenzidene for 5-10 min at room temperature (Sangon Biotech Co., Ltd, Shanghai, China).

Detection of mutations. Samples were fixed in formalin and embedded in paraffin, then sectioned as aforementioned. Mutations to genes including KRAS, epidermal growth factor receptor (EGFR), von Hippel-Lindau tumor suppressor (VHL) and vascular epidermal growth factor receptor (VEGFR), were detected by Suzhou MicroDiag Biomedicine Company Co., Ltd. (Suzhou, China) using an Taqman-Amplification refractory mutation system (Applied Biosystems; Thermo Fisher Scientific, Inc., Waltham, MA, USA).

\section{Results}

Of the 394 cases of pancreatic-occupying lesions, 4 patients $(1.02 \%)$ were diagnosed with pancreatic metastases between January 2013 and July 2016 (Table I), including 3 males and 1 female. At the time of pancreatic metastasis diagnosis, the mean age of the patients was 52.5 years (range, 43-70 years).

In 2 cases, the pancreatic metastases were synchronous with the primary tumor, whereas the other 2 cases were metachronous, with latency periods of 17 and 24 months, respectively, between the primary tumor and the metastases. The primary tumors were lung cancer in 1 case, renal cell cancer in 2 cases, and gastric cancer in 1 case. Regarding the focality of the pancreatic metastases, 3 patients exhibited unifocal lesions ( 1 in the tail and 2 in the head of the pancreas), and 1 had multifocal lesions, with 3 lesions located in the pancreatic head, 4 in the body, and 2 in the tail of the pancreas.

Only 2 patients were diagnosed based on abdominal discomfort, whereas the other 2 were diagnosed during follow-up after primary tumor resection. Upon diagnosis, 3 patients were treated by various metastasectomy methods, including distal pancreatectomy (Case 1), pancreaticoduodenectomy (Case 2), and pancreatic tumor enucleation (Case 3). Case 4 underwent EUS-FNA and pathological diagnosis. Mutation detection was performed in 3 patients to obtain precise mutation information. The detection of mutations in Case 1 revealed KRAS Gly12(-), EGFR E19Del(+) and EGFR INS(-), whereas Case 2 and Case 4 exhibited $V H L(+)$ and VEGFR2 increase.

All 4 patients were diagnosed with pancreatic metastases by CT imaging or MRI, and confirmed by pathological staining and immunohistochemistry (Figs. 1-4). MRI revealed pancreatic metastasis as a unifocal hypo-signal lesion in the tail of the pancreas on T1WI image, hematoxylin and eosin staining indicated poorly-differentiated carcinoma, and immunohistochemical staining showed Ki-67 expression (70\%) in pancreatic metastasis tissues (Fig. 1). As shown in Fig. 2, CT showed pancreatic metastasis as a unifocal hypervascular lesion in the head of the pancreas, hematoxylin and eosin staining indicated metastatic clear cell carcinoma, and immunohistochemical staining showed positive expression of CK protein in pancreatic metastasis tissues. CT showed pancreatic metastasis presented as unifocal lesion in the head of the pancreas, hematoxylin and eosin staining showed moderately-differentiated adenocarcinoma, and immunohistochemical staining showed Ki-67 expression (15\%) in pancreatic metastasis tissues (Fig. 3). As shown in Fig. 4, CT showed pancreatic metastases as multifocal lesions in the pancreas, hematoxylin and eosin staining showed metastatic clear cell carcinoma, and immunohistochemical staining showed positive expression of CK protein in pancreatic metastasis tissues. Molecular targeted drugs, according to the mutation information, were prescribed for 2 patients (Cases 2 and 4; sunitinib, $50 \mathrm{mg}$, orally, once per day, 4/2 dosage regimen). Sunitinib is a small-molecule inhibitor of several receptor tyrosine kinases relevant to tumor angiogenesis, including the vascular endothelial growth factor (VEGF) receptor $(12,13)$. Chemotherapy was administered to 1 patient [Case 3; day 1: oxaliplatin $150 \mathrm{mg}+\mathrm{CF} 0.5 \mathrm{iv}+$ fluorouracil (5-FU) $0.5 \mathrm{iv}$, 


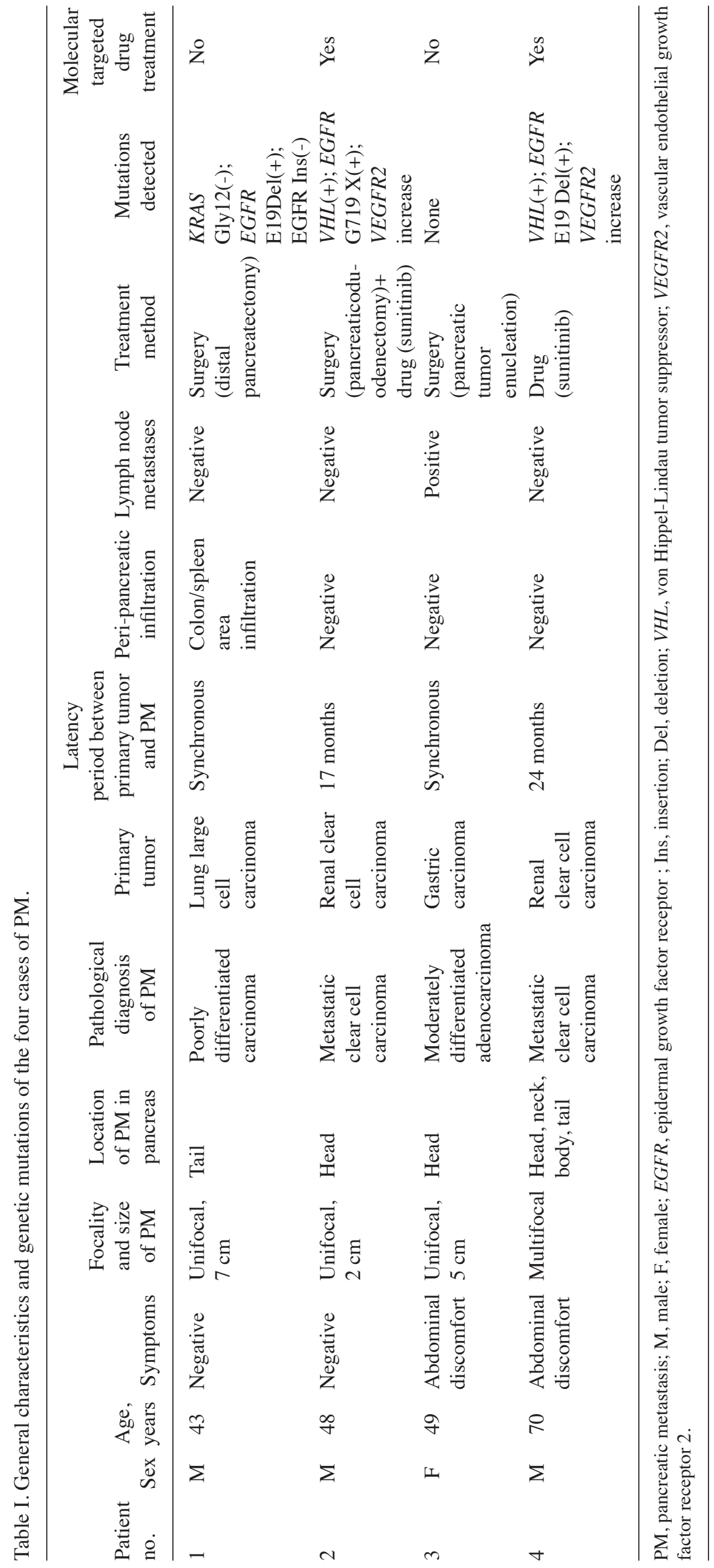



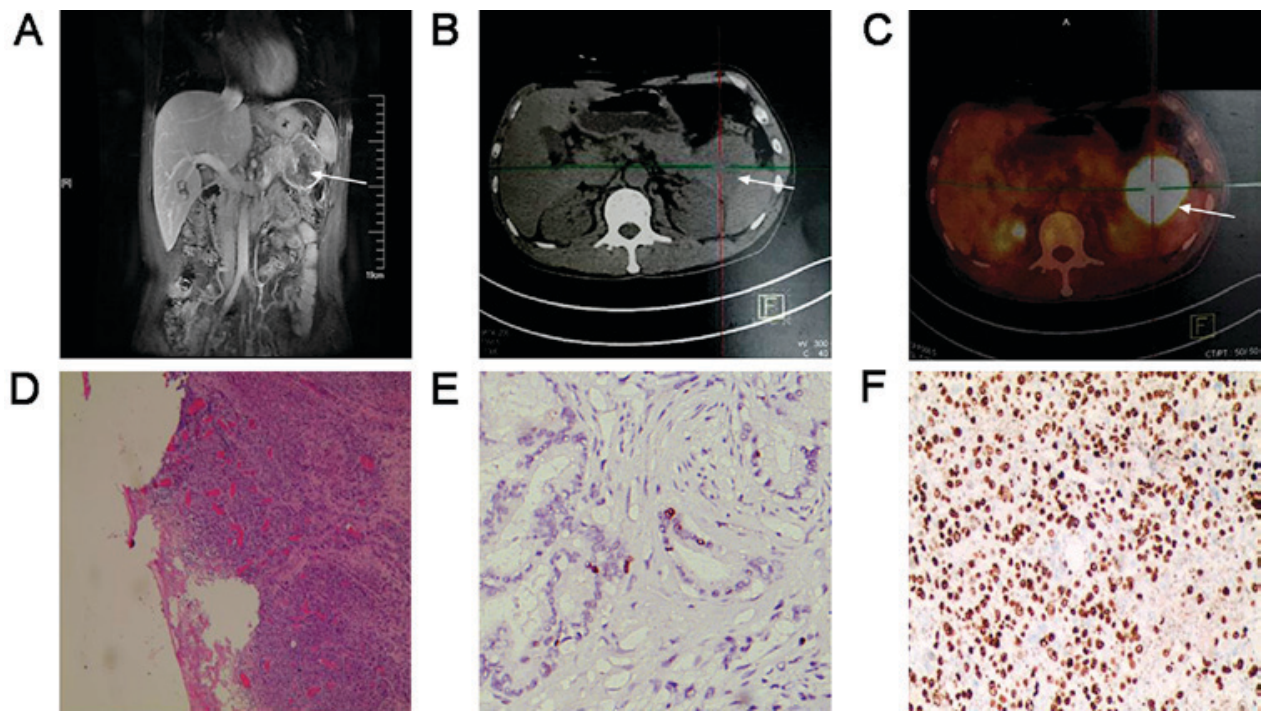

Figure 1. MRI, PET-CT and pathological findings of Case 1. (A) MRI revealed pancreatic metastasis as a unifocal lesion in the tail of pancreas (white arrow). (B and C) PET-CT images. (D) Pathological hematoxylin and eosin staining indicated poorly differentiated carcinoma (original magnification, $x 40$ ). (E and F) Immunohistochemical staining of Ki-67 (dark brown; original magnification, x100): (E) Ki-67 index of 2\% in non-tumor pancreatic tissues; (F) Ki-67 index of 70\% in pancreatic metastasis tissue. MRI, magnetic resonance imaging; PET-CT, positron-emission tomography-computed tomography.

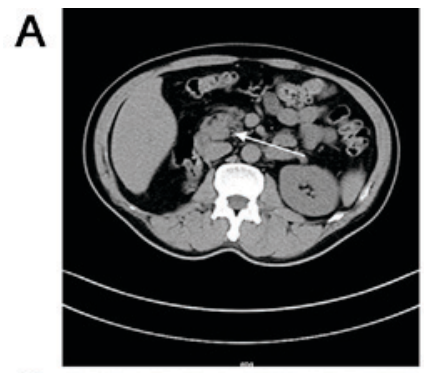

D

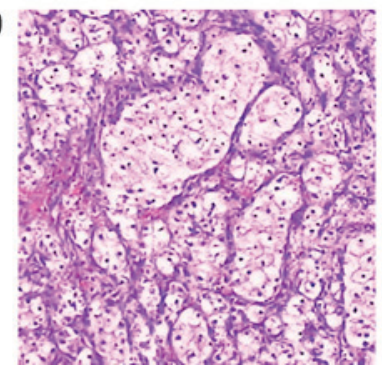

B



E

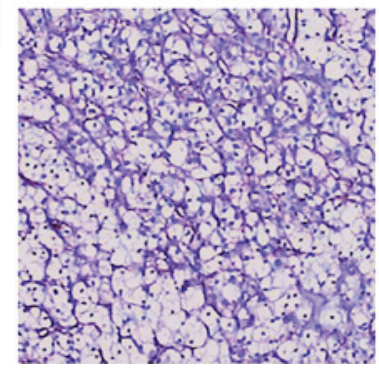

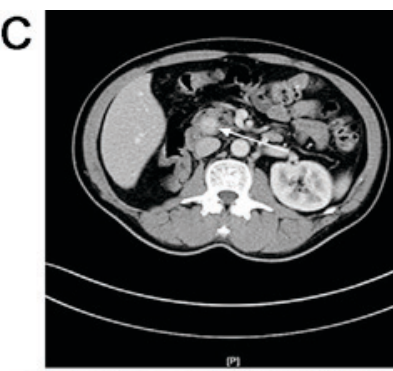

$F$

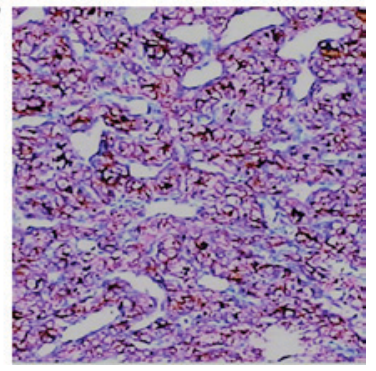

Figure 2. CT and pathological findings of Case 2. (A-C) CT showed pancreatic metastasis as a unifocal lesion in the head of pancreas (white arrow): (A) Venous phase; (B) arterial phase; (C) hepatic venous phase. (D) Pathological hematoxylin and eosin staining indicated metastatic clear cell carcinoma (original magnification, x100). (E and F) Immunohistochemical staining of cytokeratin protein (original magnification, x100): (E) negative staining in non-tumor pancreatic tissues; (F) positive staining (dark brown) in pancreatic metastasis tissue. CT, computed tomography.

followed by a $46 \mathrm{~h}$ continuous 5-FU $3.0 \mathrm{civ}$ (iv, intravenous infusion; civ, central venous infusion)]. The follow-up periods were $0.5,3,2.8$ and 0.6 year for Cases 1-4, respectively. All four patients are currently alive.

\section{Discussion}

Although metastases in the liver and lungs are well-described, the pancreas is an unusual site for metastasis. Out of all cases of pancreatic-occupying lesions, the reported incidence of pancreatic metastasis is $2-5 \%(1-5,7,14)$. In the present study, pancreatic metastases represented $1.02 \%$ of all cases of malignancies encountered in the pancreas. However, in a report of 190 autopsy cases by Adsay et al (1), 42\% patients $(81 / 190)$ exhibited metastases in the pancreas. This number is significantly higher than the generally accepted incidence rate (6). Therefore, the proportion of patients affected by pancreatic metastases is speculated to be significantly higher based on the longer lifespans and improved sensitivity of diagnostic medical examinations. As pancreatic metastases almost always occur in elderly patients and those with later-stage malignancies, certain authors have suggested that the prognosis for pancreatic metastasis is better than primary pancreatic cancer $(4,15)$. Research should be conducted to develop improved diagnostic and treatment techniques for pancreatic metastases. 


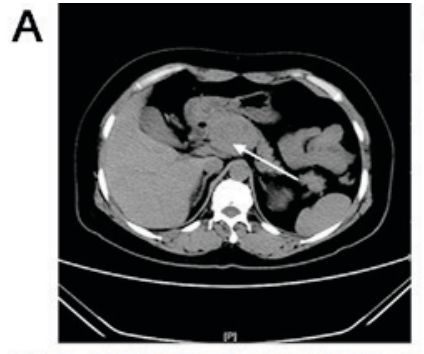

D

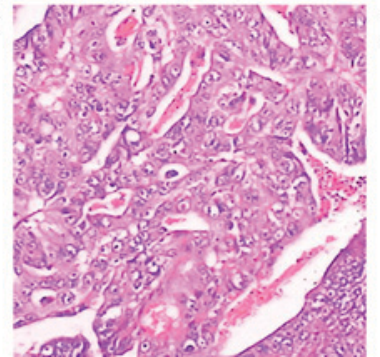

B

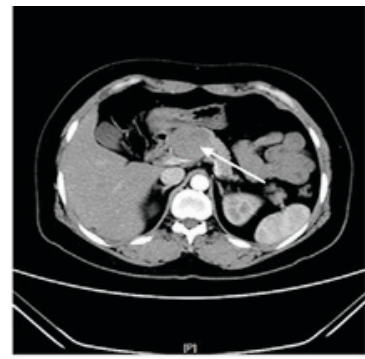

E

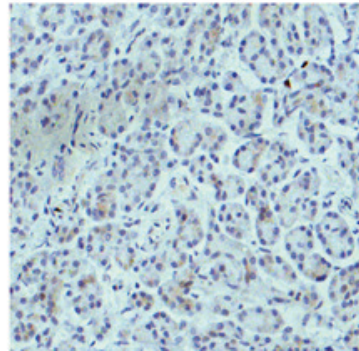

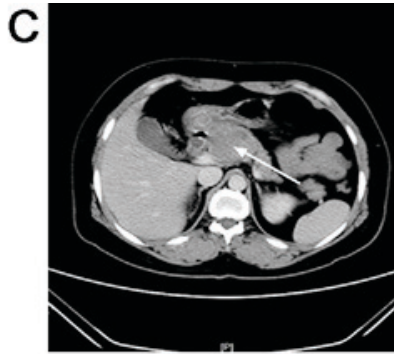

$F$



Figure 3. CT and pathological findings of Case 3. (A-C) CT showed pancreatic metastasis presented as unifocal lesion in head of pancreas (white arrow). (D) Pathological hematoxylin and eosin staining showed moderately differentiated adenocarcinoma (original magnification, $\mathrm{x} 100$ ). (E and $\mathrm{F}$ ) Immunohistochemical staining of Ki-67 (original magnification, x100): (E) Ki-67 (1\%) in non-tumor pancreatic tissues; (F) Ki-67 (15\%) in pancreatic metastasis tissues. CT, computed tomography.

A

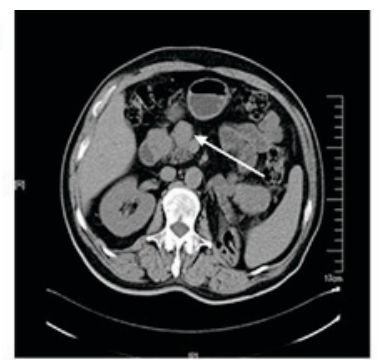

$\mathrm{D}$

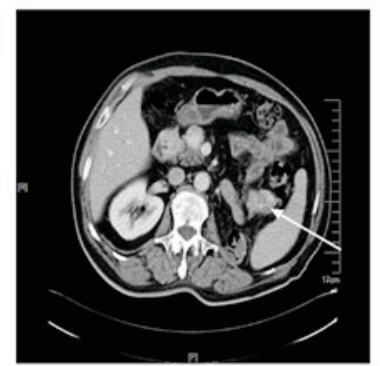

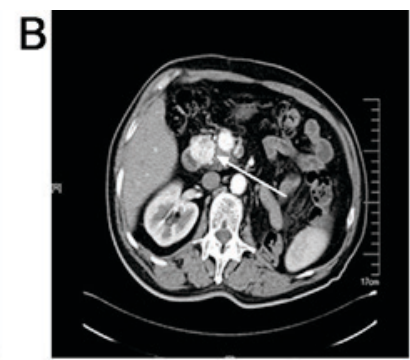

$\mathrm{E}$

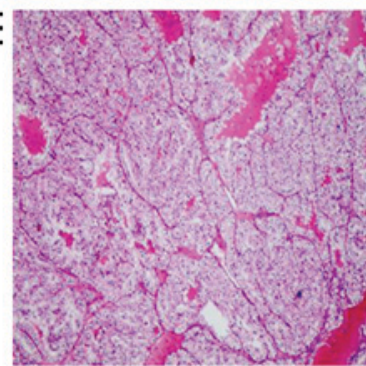

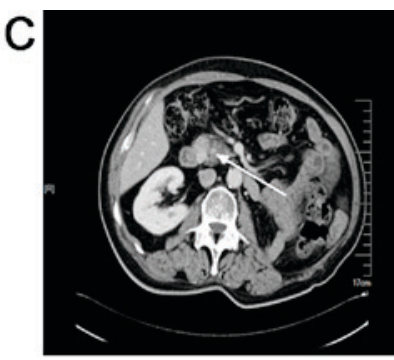

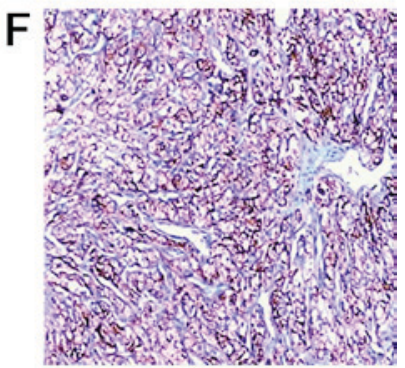

Figure 4. CT and pathological findings of Case 4. (A-C) CT imaging showed pancreatic metastases as multifocal lesions in the head of the pancreas (white arrow). (D) CT imaging revealed pancreatic metastases as multifocal lesions in the tail of the pancreas (white arrow). (E) Pathological hematoxylin and eosin staining showed metastatic clear cell carcinoma (original magnification, $\mathrm{x} 40$ ). (F) Positive immunohistochemical staining of cytokeratin (dark brown) in pancreatic metastasis tissue (original magnification, x100). CT, computed tomography.

In the present study, 3 of the 4 patients were male. However, in previously published reports, the distribution was equal between the two sexes $(6,15)$. Pancreatic metastases can occur in any part of the pancreas. Jarufe et al reported that 11 patients $(84.6 \%)$ had tumors in the head of pancreas, whereas 2 exhibited lesions in the body and tail of the pancreas (14). The majority of pancreatic metastases are unifocal (63.6\%) (7), while multifocal presentation of pancreatic metastases accounts for 20 to $45 \%(7,16)$, and diffuse-type accounts for $\sim 9 \%$ (7). As shown in a previous study of 399 patients with pancreatic metastases, the mean age at the time of diagnosis is 61.7 years (6). Following treatment of the primary tumor, pancreatic metastases have an unpredictable latency period; they may be synchronous or metachronous with the primary tumors. El Hajj et al (17) reported a case of RCC in which pancreatic metastasis was detected 29 years after diagnosis of the primary tumor. In another study, the median time between diagnosis of the primary tumor and of the initial pancreatic metastasis was 9 years (18).

Metastatic pancreatic lesions often differ from primary pancreatic lesions; $90 \%$ of primary pancreatic tumors are of ductal origin, whereas metastatic pancreatic lesions are often of epithelial or hematopoietic origin. Data from Adsay et al (1) showed that pancreatic metastases, including those arising from lung cancer, RCC and digestive cancers, were predominantly of epithelial origin (65 of 81 cases). RCC metastasis to 
the pancreas has been extensively reported in the literature, and this is considered the most common origin of pancreatic metastatic lesions (18-22). A recent literature review (18), including 582 patients with pancreatic metastases, showed that the five most common primary tumors are RCC, lung carcinoma, colorectal cancer, melanoma, and breast carcinoma, representing $80 \%$ of all cases. However, Adsay et al (1) and Tsitouridis et al (7) reported that the most common origin of metastases in the pancreas was primary lung cancer. This controversy is likely due to the different compositions between the samples. Furthermore, a study from Japan indicated stomach cancer as the most common origin of pancreatic metastases, suggesting population-based differences (23).

A proportion of patients with pancreatic metastases may experience certain non-specific symptoms, including abdominal discomfort, back pain, nausea and weight loss (24). However, the majority of patients with pancreatic metastases $(50-83 \%)$ do not exhibit any obvious symptoms, and the patient's admission to the diagnosis may appear several years after primary tumor resection (25). Metastases are usually diagnosed during follow-up studies. Therefore, in patients with pancreatic tumors and history of carcinoma, pancreatic metastasis should always be considered.

Tumor markers, including carcinoembryonic antigen (CEA) and carbohydrate antigen 19-9 (CA19-9), do not aid in the diagnosis of pancreatic metastases, although CA19-9 is a relatively sensitive indicator for primary pancreatic cancer (10). Abdominal CT can be used in the differential diagnosis of pancreatic lesions. Pancreatic metastases are usually hypervascular, whereas primary pancreatic adenocarcinoma is typically hypovascular (with the exception of endocrine pancreatic tumors, which appear as hypervascular lesions). Furthermore, compared with primary pancreatic cancer, pancreatic metastases exhibit less vascular invasion and peri-pancreatic lymphatic metastasis (7). Positron-emission tomography (PET)/CT may be advantageous in the detection of distant metastases (26). Among the 4 patients in the present study, only the patient in Case 1 underwent PET/CT. Due to its higher cost, PET/CT is not routinely used as an examination prior to surgery in clinical practice in China (7). However, as metastatic lesions may be small, it can be difficult to detect them without PET/CT (26).

Pathological analysis is the gold standard in the diagnosis of pancreatic metastases. FNA may be useful in pathological diagnosis when a specimen is difficult to remove surgically. EUS-FNA is a minimally invasive and accurate method of sampling lesions of the pancreas $(8,27)$; it has been shown to have an accuracy of $89 \%$ in diagnosing pancreatic metastases (28).

With the popularity of gene-targeted therapy, the detection of individual tumor mutations is being utilized more widely in clinical settings. The detection of mutations can provide precise pharmacogenomics-related information for individual tumor patients (29). According to the detected mutation information, doctors can classify tumors precisely, select appropriate drug treatments, and provide individualized therapy (29).

Occasionally, difficulty arises in determining the primary tumor that originated the pancreatic metastasis based solely on pathological and immunohistochemistry results; however, this difficulty may be overcome with the combination of mutation information (30). Studies have shown that thyroid transcription factor 1 (TTF-1) and napsin A are specific markers for primary lung adenocarcinoma (31,32), and that cytokeratin 20 (CK20) and caudal type homeobox 2 (CDX2) positivity supported the diagnose of primary pancreatic cancer (30); however, the positivity rate of TTF-1 and Napsin A is low. Krasinskas et al (30) reported that KRAS G12C mutations, TTF-1 and napsin A are associated with primary lung adenocarcinoma, whereas KRAS G12R mutations, CK20 and CDX2 are more common in pancreatic adenocarcinoma. Combining gene mutation information and immunohistochemical results can improve the accuracy of primary tumor diagnosis. Research has demonstrated that activating KRAS mutations are present in the majority of pancreatic ductal adenocarcinomas $(\sim 90 \%)$ and less frequently in lung adenocarcinomas $(13-23 \%)(33,34)$. EGFR gene mutations are present in $30-48 \%$ of lung adenocarcinomas and less frequently in pancreatic adenocarcinomas (2.3\%) (35). VHL gene mutations or inactivation are present in $80 \%$ of clear cell RCCs, and may promote excessive expression of VEGF, platelet-derived growth factors (PDGF), and transforming growth factor- $\alpha$ (36). Tumors are the result of accumulation of multiple mutations and their interactions (33). Oncogenic gain-of-function mutations cause excessive stimulation of the cell cycle, while loss-of-function mutations in tumor suppressor genes can result in a loss of control over this excessive activity (36). The detection of mutations in pancreatic metastases can help to confirm the primary tumor (35). In the present study, 3 patients underwent the detection of mutations. Although the immunohistochemical analysis results of Case 1 indicated TTF-1(-) and napsin A(-) expression, the combina-

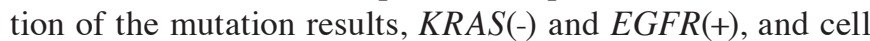
morphology led to a diagnosis of the primary tumor as a large cell lung carcinoma. Mutation detection in Cases 2 and 4 indicated $V H L(+)$, confirming the diagnosis of the primary tumor as RCC. The detection of typical mutation profiles can aid in the diagnosis of pancreatic metastases; and combining mutation information and pathology, it may be easier to confirm the diagnosis of the primary tumor.

Mutation information also has clinical value with regard to drug selection and effective prediction of pancreatic metastases. Although distant metastasis is considered a late manifestation of primary cancer, radical resection of metastases in the pancreas is the current standard treatment for pancreatic metastases in patients with no contraindication to surgery, and can improve patient survival $(11,24,37,38)$. However, for patients with pancreatic metastases and extra-pancreatic metastases, radical resection cannot be implemented.

Sunitinib is used as molecularly targeted drug for advanced metastatic clear cell RCC. The primary targets of sunitinib are VEGF receptors (VEGFRs) and PDGF receptors. In the present study, the expression of VEGFR was detected in 2 patients, which indicated that molecularly targeted drug treatment with sunitinib would be effective. Niess et al (39) compared the outcomes of patients with extra-pancreatic metastases at the time of resection, and reported a median survival time of 11 months in 8 patients with multi-organ disease. Medioni et al (13) performed a retrospective review of patients treated with sunitinib for metastatic RCC, and reported a median survival time of 20 months. Although the compositions of the two study samples were different, we speculate 
that using the molecularly targeted drug sunitinib based on mutation information may prolong the survival time of patients with unresectable pancreatic metastases and extra-pancreatic metastases. However, this hypothesis requires confirmation through further study.

In conclusion, pancreatic metastasis is rare, and its morbidity appears to be significantly increasing with the extension of human lifespan and improved sensitivity of diagnostic examinations. The majority of pancreatic metastases develop from RCC, and also commonly from lung carcinoma, colorectal cancer, melanoma and breast carcinoma. Metastases can occur several months or several years after surgery. The diagnosis of pancreatic metastases predominantly relies on $\mathrm{CT}$, pathology and immunohistochemistry. The detection of mutations has clinical value as an auxiliary test to aid diagnosis and therapy selection for pancreatic metastases. Using molecularly targeted drugs based on mutation information may prolong the survival time of patients with unresectable pancreatic metastases and extra-pancreatic metastases.

\section{Acknowledgements}

This project was supported by the Project of Key Research and Development of Jiangsu Province of China (grant no. BE2016673), the Project of Suzhou People's Livelihood Science and Technology (grant nos. SS201531 and SS201632), and Jiangsu Provincial Medical Youth Talent (grant no. QNRC2016734).

\section{References}

1. Adsay NV, Andea A, Basturk O, Kilinc N, Nassar H and Cheng JD: Secondary tumors of the pancreas: An analysis of a surgical and autopsy database and review of the literature. Virchows Arch 444: 527-535, 2004

2. Zerbi A, Ortolano E, Balzano G, Borri A, Beneduce AA and Di Carlo V: Pancreatic metastasis from renal cell carcinoma: Which patients benefit from surgical resection? Ann Surg Oncol 15: 1161-1168, 2008.

3. Roland $\mathrm{CF}$ and van Heerden JA: Nonpancreatic primary tumors with metastasis to the pancreas. Surg Gynecol Obstet 168: 345-347, 1989.

4. Faure JP, Tuech JJ, Richer JP, Pessaux P, Arnaud JP and Carretier M: Pancreatic metastasis of renal cell carcinoma: Presentation, treatment and survival. J Urol 165: 20-22, 2001.

5. Crippa S, Angelini C, Mussi C, Bonardi C, Romano F, Sartori P, Uggeri $\mathrm{F}$ and Bovo G: Surgical treatment of metastatic tumors to the pancreas: A single center experience and review of the literature. World J Surg 30: 1536-1542, 2006.

6. Adler H, Redmond CE, Heneghan HM, Swan N, Maguire D, Traynor O,Hoti E, Geoghegan JG and Conlon KC: Pancreatectomy for metastatic disease: A systematic review. Eur J Surg Oncol 40: 379-386, 2014.

7. Tsitouridis I, Diamantopoulou A, Michaelides M, Arvanity M and Papaioannou S: Pancreatic metastases: CT and MRI findings. Diagn Interv Radiol 16: 45-51, 2010.

8. Mallery JS, Centeno BA, Hahn PF, Chang Y, Warshaw AL and Brugge WR: Pancreatic tissue sampling guided by EUS, CT/US, and surgery: A comparison of sensitivity and specificity. Gastrointest Endosc 56: 218-224, 2002.

9. Chu PG, Chung L, Weiss LM and Lau SK: Determining the site of origin of mucinous adenocarcinoma: An immunohistochemical study of 175 cases. Am J Surg Pathol 35: 1830-1836, 2011.

10. Zerbi A and Pecorelli N: Pancreatic metastases: An increasing clinical entity. World J Gastrointest Surg 2: 255-259, 2010.

11. Reddy $\mathrm{S}$ and Wolfgang CL: The role of surgery in the management of isolated metastases to the pancreas. Lancet Oncol 10 287-293, 2009

12. Radulovic S and Bjelogrlic SK: Sunitinib, sorafenib and mTOR inhibitors in renal cancer. J Buon 12 (Suppl 1): S151-S162, 2007.
13. Medioni J, Choueiri TK, Zinzindohoue F, Cho D, Fournier L and Oudard S: Response of renal cell carcinoma pancreatic metastasis to sunitinib treatment: A retrospective analysis. J Urol 181: 2470-2475, 2009

14. Jarufe N, McMaster P, Mayer AD, Mirza DF, Buckels JA, Orug T, Tekin K and Bramhall SR: Surgical treatment of metastases to the pancreas. Surgeon 3: 79-83, 2005.

15. Markinez I, Jiménez R, Ruiz I, Villarreal E, Lizarazu A, Borda N, Arteaga X, Medrano MÁ, Guisasola E, Beguiristain A and Enríquez-Navascués JM: Pancreatic metastases due to renal carcinoma. Our cases and a literature review. Cir Esp 91: 90-95, 2013 (In Spanish).

16. Machado NO and Chopra P: Pancreatic metastasis from renal carcinoma managed by Whipple resection. A case report and literature review of metastatic pattern, surgical management and outcome. JOP 10: 413-418, 2009.

17. El Hajj II, LeBlanc JK, Sherman S, Al-Haddad MA, Cote GA, McHenry L and DeWitt JM: Endoscopic ultrasound-guided biopsy of pancreatic metastases: A large single-center experience. Pancreas 42: 524-530, 2013.

18. Smith AL, Odronic SI, Springer BS and Reynolds JP: Solid tumor metastases to the pancreas diagnosed by FNA: A single-institution experience and review of the literature. Cancer Cytopathol 123: 347-355, 2015.

19. Thompson LD and Heffess CS: Renal cell carcinoma to the pancreas in surgical pathology material. Cancer 89: 1076-1088, 2000.

20. Hiotis SP, Klimstra DS, Conlon KC and Brennan MF: Results after pancreatic resection for metastatic lesions. Ann Surg Oncol 9: 675-679, 2002.

21. Klein KA, Stephens DH and Welch TJ: CT characteristics of metastatic disease of the pancreas. Radiographics 18: 369-378, 1998.

22. Le Borgne J, Partensky C, Glemain P, Dupas B and de Kerviller B: Pancreaticoduodenectomy for metastatic ampullary and pancreatic tumors. Hepatogastroenterology 47: 540-544, 2000.

23. Nakamura E, Shimizu M, Itoh T and Manabe T: Secondary tumors of the pancreas: Clinicopathological study of 103 autopsy cases of Japanese patients. Pathol Int 51: 686-690, 2001.

24. Sweeney AD, Fisher WE, Wu MF, Hilsenbeck SG and Brunicardi FC: Value of pancreatic resection for cancer metastatic to the pancreas. J Surg Res 160: 268-276, 2010.

25. Ahmed S, Johnson PT, Hruban R and Fishman EK: Metastatic disease to the pancreas: Pathologic spectrum and CT patterns. Abdom Imaging 38: 144-153, 2013.

26. Song SW, Cheng JF, Liu N and Zhao TH: Diagnosis and treatment of pancreatic metastases in 22 patients: A retrospective study. World J Surg Oncol 12: 299, 2014.

27. Chen L and Brainard JA: Pancreatic metastasis from papillary thyroid carcinoma diagnosed by endoscopic ultrasound-guided fine needle aspiration: A case report. Acta Cytol 54: 640-644, 2010.

28. Ardengh JC, Lopes CV, Kemp R, Venco F, de Lima-Filho ER and dos Santos JS: Accuracy of endoscopic ultrasound-guided fine-needle aspiration in the suspicion of pancreatic metastases. Bmc Gastroenterol 13: 63, 2013.

29. Saiz M, Alvarez-Cubero MJ, Martinez-González LJ, Alvarez JC, Lorente $\mathrm{M}$ and Lorente JA: The identification of individuals through genetic testing and DNA profiling banks. Rev Derecho Genoma Hum: 157-168, 2014.

30. Krasinskas AM, Chiosea SI, Pal T and Dacic S: KRAS mutational analysis and immunohistochemical studies can help distinguish pancreatic metastases from primary lung adenocarcinomas. Mod Pathol 27: 262-270, 2014.

31. Wu J, Chu PG, Jiang Z and Lau SK: Napsin A expression in primary mucin-producing adenocarcinomas of the lung: An immunohistochemical study. Am J Clin Pathol 139: 160-166, 2013.

32. Stoll LM, Johnson MW, Gabrielson E, Askin F, Clark DP and Li QK: The utility of napsin-A in the identification of primary and metastatic lung adenocarcinoma among cytologically poorly differentiated carcinomas. Cancer Cytopathol 118: 441-449, 2010.

33. Hruban RH, van Mansfeld AD, Offerhaus GJ, van Weering DH, Allison DC, Goodman SN, Kensler TW, Bose KK, Cameron JL and Bos JL: K-ras oncogene activation in adenocarcinoma of the human pancreas. A study of 82 carcinomas using a combination of mutant-enriched polymerase chain reaction analysis and allele-specific oligonucleotide hybridization. Am J Pathol 143: 545-554, 1993. 
34. Dacic S, Shuai Y, Yousem S, Ohori P and Nikiforova M: Clinicopathological predictors of EGFR/KRAS mutational status in primary lung adenocarcinomas. Mod Pathol 23: $159-168,2010$.

35. Oliveira-Cunha M, Hadfield KD, Siriwardena AK and Newman W: EGFR and KRAS mutational analysis and their correlation to survival in pancreatic and periampullary cancer. Pancreas 41: 428-434, 2012.

36. Patard JJ, Rioux-Leclercq N, Masson D, Zerrouki S, Jouan F, Collet N, Dubourg C, Lobel B, Denis M and Fergelot P: Absence of VHL gene alteration and high VEGF expression are associated with tumour aggressiveness and poor survival of renal-cell carcinoma. Br J Cancer 101: 1417-1424, 2009.
37. Reddy S, Edil BH, Cameron JL, Pawlik TM, Herman JM, Gilson MM, Campbell KA, Schulick RD, Ahuja N and Wolfgang CL: Pancreatic resection of isolated metastases from nonpancreatic primary cancers. Ann Surg Oncol 15: 3199-3206, 2008.

38. Strobel O, Hackert T, Hartwig W, Bergmann F, Hinz U, Wente MN, Fritz S, Schneider L, Büchler MW and Werner J: Survival data justifies resection for pancreatic metastases. Ann Surg Oncol 16: 3340-3349, 2009.

39. Niess H, Conrad C, Kleespies A, Haas F, Bao Q, Jauch KW, Graeb C and Bruns CJ: Surgery for metastasis to the pancreas: Is it safe and effective? J Surg Oncol 107: 859-864, 2013. 